\title{
Desempenho de cordeiros provenientes de ovelhas de diferentes grupos genéticos e que foram submetidas ao flushing alimentar
}

\section{Performance of lambs out of ewes from different genetic groups and submitted to flushing}

\author{
Edson Luis de Azambuja Ribeiro ${ }^{1 *}$; Rinaldo Masato Mori²; \\ Ivone Yurika Mizubuti ${ }^{1}$; Leandro das Dores Ferreira da Silva ${ }^{1}$; \\ Odimári Pricila Pires do Prado ${ }^{1}$; Elzânia Sales Pereira ${ }^{3}$; Andréa Pereira Pinto ${ }^{3}$; \\ Patrícia Guimarães Pimentel ${ }^{3}$; Ricardo Fávero ${ }^{4}$
}

\begin{abstract}
Resumo
Este estudo teve por objetivos avaliar o desempenho produtivo de cordeiros até o desmame, filhos de ovelhas de diferentes grupos genéticos e submetidas a diferentes suplementações alimentares antes e durante a estação de monta. Foram utilizados 126 cordeiros, sendo 11 1/2 Corriedale $+1 / 2$ Texel, 73 Hampshire Down, 26 Ile de France e 16 Suffolk, nascidos em dois anos consecutivos, provenientes de ovelhas submetidas a três tratamentos: T1 - sem suplementação, T2 - suplementação com 600 g de milho triturado por dia, e T3 - suplementação com $600 \mathrm{~g}$ de uma mistura de $75 \%$ de milho e $25 \%$ de farelo de soja; mantidas em pastagem de Coast-cross. Os dados de pesos e ganhos de peso foram analisados individualmente e por prole (soma dos gêmeos). O flushing não influenciou os pesos e ganhos de peso. Cordeiros gêmeos, na análise individual, apresentaram pesos e ganhos de pesos menores do que cordeiros únicos, porém, na análise por prole, na soma dos gêmeos observou-se maiores pesos e ganhos do que em cordeiros únicos. Verificaram-se diferenças nos pesos e ganhos de peso por influência dos grupos genéticos e por sexo, onde os machos foram mais pesados e ganharam mais peso do que as fêmeas. Cordeiros gêmeos apresentaram maior mortalidade que cordeiros únicos. Apesar do flushing prover maior produção de cordeiros, não se verificou um retorno econômico considerável. Conclui-se que oflushing alimentar não influencia o desempenho dos cordeiros, e que cordeiros gêmeos apresentam maior taxa de mortalidade que cordeiros únicos, sendo o flushing alimentar pouco viável economicamente.
\end{abstract}

Palavras-chave: Ganho de peso, gêmeos, ovinos, raças, suplementação alimentar

\begin{abstract}
The objectives of this study were to analyze the productive performance of lambs, out of ewes of different genetic groups and submitted to different feeding managements before and during the mating season, from birth until weaning. One hundred and twenty-six lambs were used, from the genetic
\end{abstract}

${ }^{1}$ Profs. Drs., Dept ${ }^{\circ}$ de Zootecnia, Universidade Estadual de Londrina, UEL, Londrina, PR. E-mail: elar@uel.br; mizubuti@uel.br; leandro@uel.br; odimari@uel.br

${ }^{2}$ Pesquisador Dr., Ministério da Agricultura, Pecuária e Abastecimento, MAPA, Dionísio Cerqueira, SC. E-mail: morivet2002@ yahoo.com.br

3 Prof $^{\text {s }}$ Dr $^{\text {as }}$, Dept ${ }^{\circ}$ de Zootecnia, Universidade Federal do Ceará, UFC, Fortaleza, CE. E-mail: elzania@hotmail.com; deiapp@ hotmail.com; pgpimentel@hotmail.com

${ }^{4}$ Discente do Programa de Pós-Graduação em Ciência Animal, UEL, Londrina, PR. E-mail: ricardo.mvet@yahoo.com.br

* Autor para correspondência 
groups $1 / 2$ Corriedale $+1 / 2$ Texel (11), Hampshire Down (73), Ile de France (26) and Suffolk (16), born in two consecutive years. The ewes were submitted to three feeding managements: T1 - without supplementation, T2 - supplemented with $600 \mathrm{~g}$ /day of corn grain, and T3 - supplemented with $600 \mathrm{~g} /$ day of a ration containing $75 \%$ of corn and $25 \%$ of soybean meal. Body weight and weight gain were analysed individually and by litter size. Flushing did not influence weight and weight gain of lambs. Twin lambs, in the individual analysis, showed lower body weight and weight gain compared to single lambs, but in the analysis by litter size, the sum of the twin lambs showed greater body weight and weight gain compared to single lambs. Differences in body weight and weight gain were observed among the genetic groups and between the sexes, where males were heavier and presented greater weight gain. Twin lambs showed higher mortality than single lambs. Although flushing can provide greater number of lambs, a considerable profit was not verified. It is concluded that flushing do not influence in the performance of lambs, and that twin lambs show higher mortality than single ones, and flushing is not profitable.

Key words: Breeds, feed supplementation, sheep, twins, weight gain

\section{Introdução}

A produção de ovinos para corte é uma atividade em franco crescimento. Sendo que a produção de carne tem íntima relação com o desempenho reprodutivo das ovelhas, e este fator muitas vezes limita uma maior produtividade do rebanho ovino.

A herdabilidade das características que afetam o desempenho reprodutivo dos ovinos apresenta valores que variam de 3 a 17\% (MOHAMMADI; SHAHRBABAK; SHAHRBABAK, 2012; ZISHIRI et al., 2013). Portanto, seleção para fertilidade apresenta resultados significativos apenas a longo prazo. Devido a isto, pesquisas levando em consideração a melhoria de aspectos ambientais, visando aumentos nas taxas reprodutivas, e consequentemente maior produção de animais para abate, devem ser desenvolvidas.

Segundo Scaramuzzi e Radford (1983), para se melhorar as taxas de parição e prolificidade, devese aumentar a taxa ovulatória. Sobre esta influem muitos fatores, dos quais a nutrição é um dos mais importantes. A suplementação alimentar antes e durante o período de acasalamento (flushing) possibilita aumentos significativos nos parâmetros reprodutivos das ovelhas, através do aumento da taxa ovulatória (NOTTLE et al., 1997; BRANCA et al., 2000; LASSOUED et al., 2004; NAQVI; SEJIAN; KARIM, 2012), e através do aumento da incidência de partos gemelares (NOTTLE et al.,
1997; LASSOUED et al., 2004).

A prática do flushing determina maior ocorrência de partos gemelares, porém, os cordeiros nascidos de partos gemelares possuem menor peso ao nascimento e esse fato está diretamente relacionado com maior taxa de mortalidade entre cordeiros gêmeos comparado com cordeiros únicos. Além disso, os cordeiros provenientes de partos gemelares possuem menor peso ao desmame, quando analisados individualmente. Roda e Otto (1990), avaliando o flushing em ovelhas Ideal e Corriedale, verificaram, através de uma análise simplificada, que esta prática foi anti-econômica, pois o investimento em ração por cabeça de cordeiro excedente superou claramente o seu valor de mercado.

Sormunen-Cristian e Jauhiainen observaram que a resposta ao flushing, depende da idade da ovelha, onde animais muito jovens (1 ano de idade) ou velhos (7 e 8 anos de idade) não respondem a este tratamento. Os autores concluíram que, de uma maneira geral, o ganho em produção de cordeiros pelo flushing em ovelhas Finnish Landrace foi pequeno.

Peeters, Kox e Van Isterdael (1996) citaram que maiores taxas de crescimento de cordeiros até o abate é um dos fatores que determinam maiores retornos para o produtor. Esses autores, estudando animais three-cross $(1 / 4$ Flemish $+1 / 4$ Suffolk $+1 / 2$ Texel $)$, observaram ainda que pesos ao nascimento, época 
de nascimento, tamanho da prole, idade da ovelha e sexo do cordeiro têm influência significativa sobre a taxa de crescimento pós-natal.

Como observado na literatura citada, o efeito do flushing no aumento de partos gemelares é controverso. Da mesma maneira, onde houve resposta positiva no aumento da produção de cordeiros, pouco se discute sobre a viabilidade econômica desta prática. Portanto, os objetivos deste trabalho foram acompanhar o desempenho produtivo (peso, ganho de peso e mortalidade) de cordeiros, provenientes de ovelhas submetidas a diferentes suplementações alimentares antes e durante o acasalamento, desde o nascimento até o desmame (70 dias) e analisar de maneira prática a viabilidade econômica deste manejo.

\section{Material e Métodos}

O experimento foi conduzido na Fazenda Escola da Universidade Estadual de Londrina, situada no município de Londrina, Paraná (Lat. S. 23²0'10" e Long. W. 5109'15"), com 610 metros de altitude em relação ao nível do mar, temperatura ambiente média anual de $20,6^{\circ} \mathrm{C}$, precipitação média anual de $1.439,8 \mathrm{~mm}$, sendo máxima em janeiro $(201,4 \mathrm{~mm}$ em média) e mínima em junho (56,5 mm em média) (MAACK, 1981).

Foram utilizados 126 cordeiros, sendo 11 1/2 Corriedale + 1/2 Texel, 73 Hampshire Down, 26 Ile de France e 16 Suffolk. Os animais Hampshire Down, Ile de France e Suffolk possuíam grau sanguíneo variado, sendo no mínimo $3 / 4$ de sangue. Esses animais nasceram nos anos de 2001 (70 cordeiros) e 2002 (56 cordeiros), e eram provenientes de ovelhas distribuídas ao acaso, segundo grupo genético e idade, em três grupos de suplementação antes e durante a estação de monta: T1 - sem suplementação, T2 - suplementadas com $600 \mathrm{~g}$ de grão de milho triturado por dia, durante 60 dias, e T3 - suplementadas com $600 \mathrm{~g}$ por dia de uma mistura concentrada constituída de $75 \%$ de grão de milho triturado e $25 \%$ de farelo de soja, durante 60 dias. A suplementação alimentar iniciou em 01 de março nos dois anos experimentais (15 dias antes do início da estação de monta) e persistiu até 30 de abril, totalizando 60 dias de suplementação em cada ano avaliado. Durante o dia os animais foram mantidos em pastagem de Coast-cross (Cynodon dactylon (L.) Pers) em sistema rotacionado, com água e sal mineralizado à vontade. Resultados de desempenho das ovelhas foram apresentados por Mori et al. (2006).

Nos dois anos experimentais, as estações de monta iniciaram em 15 de março e finalizaram em 30 de abril, totalizando 45 dias. Foi utilizada a monta natural, em que as ovelhas dos grupos genéticos Hampshire Down, Ile de France e Suffolk foram acasaladas com carneiros de suas respectivas raças, enquanto que as ovelhas Corriedale foram acasaladas com carneiro Texel. Todos os animais foram desverminados ao início da suplementação alimentar e, posteriormente, de acordo com a contagem de ovos por grama de fezes.

A partir da segunda quinzena de maio até o mês de novembro as ovelhas passaram a receber silagem de milho (ano de 2001) ou sorgo (ano de 2002), fornecida em comedouros em curral de manejo, todos os dias à tarde. As ovelhas paridas, a partir do parto, receberam cerca de $250 \mathrm{~g}$ por cabeça por dia de milho triturado junto com a silagem de milho ou sorgo. Os cordeiros permaneceram com suas respectivas mães até o desmame, que ocorreu aos 70 dias de idade.

Foram realizadas análises químicas e bromatológicas para mensurar a composição em matéria seca, proteína bruta, extrato etéreo, fibra bruta, matéria mineral, fibra em detergente neutro e fibra em detergente ácido através dos métodos de Weende e Van Soest (SILVA; QUEIRÓZ, 2002) das pastagens, dos suplementos alimentares e das silagens de milho e sorgo. Os resultados dessas análises são apresentados na Tabela 1. Os resultados do pasto, do milho grão e do farelo de soja são médias dos dois anos experimentais. 
Tabela 1. Composição química-bromatológica dos alimentos utilizados.

\begin{tabular}{lcccccc}
\hline \multicolumn{1}{c}{ Alimento } & MS (\%MN) & PB (\%MS) & EE (\%MS) & FDN (\%MS) & FDA (\%MS) & MM (\%MS) \\
\hline Coast-cross & 42,73 & 10,46 & 1,45 & 79,36 & 37,36 & 7,81 \\
Silagem de milho & 31,45 & 6,59 & 2,21 & 63,25 & 31,57 & 4,95 \\
Silagem de sorgo & 30,21 & 7,52 & 2,09 & 68,47 & 33,52 & 5,41 \\
Milho grão & 90,07 & 9,06 & 4,52 & - & - & 6,52 \\
Farelo de soja & 90,27 & 46,21 & 0,88 & - & - & 5,45 \\
\hline
\end{tabular}

MN: matéria natural; MS: matéria seca; PB: proteína bruta; EE: extrato etéreo; FDN: fibra em detergente neutro; FDA: fibra em detergente ácido.

Fonte: Elaboração dos autores.

A avaliação econômica foi realizada, levandose em consideração somente a suplementação alimentar das ovelhas antes e durante a estação de monta, considerando os cordeiros provenientes de ovelhas sem suplementação com custo zero. Procedeu-se a separação dos cordeiros com 70 dias de idade, por tratamento a que suas mães foram submetidas, somando-se o peso total de cada tratamento. Para validar a comparação entre os tratamentos, extrapolou-se os dados obtidos em cada tratamento para um rebanho de 100 ovelhas, tanto para peso dos cordeiros aos 70 dias de idade, quanto para suplementos consumidos durante o flushing alimentar.

Os dados coletados foram: pesos dos cordeiros ao nascimento e ao desmame (70 dias) e mortalidade dos cordeiros até o desmame. Os dados foram submetidos à análise de variância através do pacote estatístico SAS (SAS, 1994). Nas análises de pesos e ganhos de peso dos cordeiros foram levados em consideração no modelo final os efeitos do flushing (tratamento), ano (2001 e 2002), idade da ovelha (4, 6 e 8 dentes) e grupo racial ( $1 / 2$ Corriedale $+1 / 2$ Texel, Hampshire Down, Ile de France e Suffolk). Por não apresentarem significância, as interações duplas entre os efeitos foram desconsideradas do modelo estatístico final. Os dados de mortalidade nos diferentes tratamentos, anos, idades e grupos raciais foram analisados pelo teste do qui-quadrado. As diferenças entre médias foram testadas pelo teste t.

\section{Resultados e Discussão}

Pelos dados apresentados na Tabela 2, analisados por prole (somando-se os pesos dos gêmeos), e na Tabela 3, analisados individualmente, verifica-se que a suplementação alimentar das ovelhas não interferiu nos pesos ao nascimento dos cordeiros. Isto está de acordo com os resultados obtidos por Bramley, Denehy e Newton (1976).

A suplementação das mães imediatamente antes e durante o período de acasalamento não afetou o desempenho dos filhos, provavelmente porque após o final do acasalamento até o nascimento dos cordeiros, ou seja, durante o período de gestação, as ovelhas dos dois tratamentos que foram suplementadas, e que chegaram mais pesadas no final do acasalamento, posteriormente perderam peso, enquanto que as não suplementadas mantiveram o peso durante a gestação. Estas mudanças de pesos anularam as diferenças entre as ovelhas dos três tratamentos (MORI et al., 2006). 
Tabela 2. Médias $(\mathrm{kg})$ de pesos \pm erros-padrão de cordeiros ao nascimento e ao desmame (70 dias) e ganho médio diário até o desmame, analisados por prole (soma do gêmeos).

\begin{tabular}{lccc}
\hline \multicolumn{1}{c}{ Efeito } & Peso ao nascimento & Peso ao desmame & Ganho médio diário \\
\hline Tratamento $^{1}$ & & & \\
$\mathrm{~T} 1$ & $4,23 \pm 0,27$ & $19,49 \pm 1,05$ & $0,215 \pm 0,014$ \\
$\mathrm{~T} 2$ & $4,83 \pm 0,26$ & $20,01 \pm 0,97$ & $0,217 \pm 0,012$ \\
$\mathrm{~T} 3$ & $4,53 \pm 0,26$ & $18,84 \pm 0,93$ & $0,205 \pm 0,012$ \\
Pr $>\mathrm{F}$ & 0,1693 & 0,5835 & 0,6990 \\
\hline Tipo de parto & & & \\
Simples & $3,87 \pm 0,20 \mathrm{~b}$ & $17,81 \pm 0,74 \mathrm{~b}$ & $0,198 \pm 0,010 \mathrm{~b}$ \\
Gemelar & $5,18 \pm 0,27 \mathrm{a}$ & $21,09 \pm 1,02 \mathrm{a}$ & $0,226 \pm 0,013 \mathrm{a}$ \\
Pr $>$ F & 0,0001 & 0,0028 & 0,0433 \\
\hline Idade da mãe & & & \\
4 dentes & $4,05 \pm 0,32 \mathrm{~b}$ & $18,34 \pm 1,18$ & $0,203 \pm 0,015$ \\
6 dentes & $4,61 \pm 0,33 \mathrm{ab}$ & $20,19 \pm 1,17$ & $0,222 \pm 0,015$ \\
8 dentes & $4,93 \pm 0,20 \mathrm{a}$ & $19,82 \pm 0,78$ & $0,212 \pm 0,010$ \\
Pr $>$ F & 0,0489 & 0,4316 & 0,6489 \\
\hline Grupo genético & & & \\
1/2 Corriedale $+1 / 2$ Texel & $3,96 \pm 0,49$ & $18,01 \pm 1,76 \mathrm{~b}$ & $0,201 \pm 0,023 \mathrm{a}$ \\
Hampshire Down & $4,91 \pm 0,19$ & $20,22 \pm 0,70 \mathrm{a}$ & $0,218 \pm 0,009 \mathrm{a}$ \\
Ile de France & $4,42 \pm 0,31$ & $16,73 \pm 1,16 \mathrm{~b}$ & $0,177 \pm 0,015 \mathrm{~b}$ \\
Suffolk & $4,82 \pm 0,39$ & $22,84 \pm 1,61 \mathrm{a}$ & $0,254 \pm 0,021 \mathrm{a}$ \\
Pr $>$ F & 0,1809 & 0,0132 & 0,0252 \\
\hline Ano & & & $0,212 \pm 0,011$ \\
2001 & $4,48 \pm 0,22$ & $19,41 \pm 0,83$ & $0,213 \pm 0,012$ \\
2002 & $4,57 \pm 0,25$ & 0,9309 & 0,9691 \\
Pr $>$ F & 0,7438 & & \\
\hline & & &
\end{tabular}

a,b: Médias seguidas de letras diferentes na coluna, para o mesmo efeito, diferem significativamente.

${ }^{1}$ Tratamento oferecido as mães antes e durante a monta, sendo T1: Sem suplementação; T2: Suplementação com milho triturado; T3: Suplementação com farelo de soja e milho triturado (25:75).

Fonte: Elaboração dos autores.

Diferenças no desempenho pré-desmame de cordeiros são observadas quando as mães chegam em melhores condições corporais ao parto, parindo cordeiros mais pesados, e produzindo mais leite durante a lactação (CASTRO et al., 2012ab, 2013).

Em relação ao tipo de parto, quando se analisou individualmente, os cordeiros oriundos de partos gemelares foram mais leves do que cordeiros únicos desde o nascimento até o desmame. Por outro lado, quando se analisou o peso da prole, os pesos dos gêmeos foram superiores. Esses resultados concordam em parte com os achados por Mexia et al. (2004), que também verificaram que cordeiros nascidos como gêmeos foram mais leves do que os únicos, porém quando comparam a soma da prole, o peso total dos gêmeos foi similar ao dos nascidos como únicos do nascimento aos 60 dias de idade.

Ovelhas de oito dentes pariram proles mais pesadas do que ovelhas de quatro dentes, com as ovelhas de seis dentes ocupando posição intermediária. Porém, quando se analisou os cordeiros individualmente não se verificou diferença entre as idades no que se refere ao peso 
ao nascimento $(\mathrm{P}=0,0568)$. Ao desmame não houve diferença significativa para peso dos cordeiros entre as diferentes idades das ovelhas. Esses resultados concordam com os reportados por Castro et al. (2012b), que não observaram diferenças para pesos ao nascimento e ao desmame em cordeiros filhos de ovelhas com dois a oito dentes. Porém, esses autores observaram diferenças no ganho de peso médio diário, onde cordeiros filhos de ovelhas de quatro e seis dentes apresentaram maiores médias do que cordeiros filhos de ovelhas mais jovens (2 dentes) e mais velhas (8 dentes).

Tabela 3. Médias $(\mathrm{kg})$ de pesos \pm erros-padrão de cordeiros ao nascimento e ao desmame (70 dias) e ganho médio diário até o desmame, analisados individualmente.

\begin{tabular}{|c|c|c|c|}
\hline Efeito & Peso ao nascimento & Peso ao desmame & Ganho médio diário \\
\hline \multicolumn{4}{|l|}{ Tratamento $^{1}$} \\
\hline $\mathrm{T} 1$ & $3,10 \pm 0,17$ & $14,38 \pm 0,64$ & $0,159 \pm 0,008$ \\
\hline $\mathrm{T} 2$ & $3,43 \pm 0,15$ & $14,90 \pm 0,59$ & $0,164 \pm 0,008$ \\
\hline $\mathrm{T} 3$ & $3,21 \pm 0,16$ & $13,94 \pm 0,57$ & $0,153 \pm 0,007$ \\
\hline $\operatorname{Pr}>\mathrm{F}$ & 0,2361 & 0,4150 & 0,5658 \\
\hline \multicolumn{4}{|l|}{ Tipo de parto } \\
\hline Simples & $3,95 \pm 0,13 a$ & $17,83 \pm 0,49 \mathrm{a}$ & $0,198 \pm 0,006 \mathrm{a}$ \\
\hline Gemelar & $2,55 \pm 0,15 b$ & $10,98 \pm 0,56 b$ & $0,120 \pm 0,007 b$ \\
\hline $\operatorname{Pr}>F$ & 0,0001 & 0,0001 & 0,0001 \\
\hline \multicolumn{4}{|l|}{ Idade da mãe } \\
\hline 4 dentes & $2,96 \pm 0,21$ & $14,05 \pm 0,76$ & $0,157 \pm 0,010$ \\
\hline 6 dentes & $3,27 \pm 0,20$ & $14,47 \pm 0,71$ & $0,160 \pm 0,009$ \\
\hline 8 dentes & $3,51 \pm 0,12$ & $14,71 \pm 0,46$ & $0,159 \pm 0,006$ \\
\hline $\operatorname{Pr}>\mathrm{F}$ & 0,0568 & 0,7395 & 0,9801 \\
\hline \multicolumn{4}{|l|}{ Grupo genético } \\
\hline $1 / 2$ Corriedale $+1 / 2$ Texel & $2,84 \pm 0,29 b$ & $13,03 \pm 1,03 b c$ & $0,146 \pm 0,013 b c$ \\
\hline Hampshire Down & $3,59 \pm 0,12 \mathrm{a}$ & $15,08 \pm 0,42 \mathrm{ab}$ & $0,164 \pm 0,005 \mathrm{ab}$ \\
\hline Ile de France & $3,16 \pm 0,19 b$ & $12,77 \pm 0,71 \mathrm{c}$ & $0,137 \pm 0,009 c$ \\
\hline Suffolk & $3,39 \pm 0,24 \mathrm{a}$ & $16,76 \pm 1,00 \mathrm{a}$ & $0,188 \pm 0,013 \mathrm{a}$ \\
\hline $\operatorname{Pr}>\mathrm{F}$ & 0,0291 & 0,0035 & 0,0123 \\
\hline \multicolumn{4}{|l|}{ Sexo } \\
\hline Macho & $3,46 \pm 0,14 \mathrm{a}$ & $15,37 \pm 0,51 \mathrm{a}$ & $0,169 \pm 0,007 \mathrm{a}$ \\
\hline Fêmea & $3,03 \pm 0,14 b$ & $13,45 \pm 0,54 \mathrm{~b}$ & $0,148 \pm 0,007 \mathrm{~b}$ \\
\hline $\operatorname{Pr}>\mathrm{F}$ & 0,0092 & 0,0025 & 0,0097 \\
\hline \multicolumn{4}{|l|}{ Ano } \\
\hline 2001 & $3,27 \pm 0,13$ & $14,72 \pm 0,50$ & $0,163 \pm 0,007$ \\
\hline 2002 & $3,22 \pm 0,15$ & $14,09 \pm 0,54$ & $0,155 \pm 0,007$ \\
\hline $\operatorname{Pr}>\mathrm{F}$ & 0,7913 & 0,2997 & 0,3194 \\
\hline
\end{tabular}

a,b,c: Médias seguidas de letras diferentes na coluna, para o mesmo efeito, diferem significativamente.

${ }^{1}$ Tratamento oferecido as mães antes e durante a monta, sendo T1: Sem suplementação; T2: Suplementação com milho triturado; T3: Suplementação com farelo de soja e milho triturado (25:75).

Fonte: Elaboração dos autores. 
Ovelhas dos diferentes grupamentos genéticos pariram proles com pesos similares, porém a análise individual mostrou que ovelhas Hampshire Down pariram cordeiros mais pesados do que as Corriedale (cordeiros $1 / 2$ Corriedale $+1 / 2$ Texel) e Ile de France. Na análise por prole, cordeiros Suffolk e Hampshire Down apresentaram pesos similares até o desmame, o mesmo ocorrendo na análise individual. $\mathrm{Na}$ avaliação individual ocorreu um comportamento bastante parecido, sendo os cordeiros Ile de France e $1 / 2$ Corriedale $+1 / 2$ Texel mais leves ao desmame nas duas análises.

Cordeiros machos nasceram e mantiveramse mais pesados que as fêmeas até o desmame (Tabela 3). Sendo que os machos nasceram entorno de $14 \%$ mais pesados do que as fêmeas, e esta diferença percentual se manteve no desmame. Esse resultado foi similar aos observados por Koritiaki et al. (2012) e por Castro et al. (2012b, 2013). De acordo com Schanbacher, Crouse e Ferrell (1980), os machos não castrados apresentam maior ganho de peso em função principalmente do hormônio testosterona. Sendo que este hormônio provoca uma maior deposição de massa muscular. Os resultados do presente trabalho diferem dos encontrados por Mexia et al. (2004) e por Koritiaki et al. (2013), que não observaram diferenças nos pesos ao nascimento e ao desmame em cordeiros Santa Inês e cruzados Santa Inês. Esta falta de diferença entre os sexos pode estar relacionada a pouca idade dos animais no desmame (60 e 70 dias respectivamente), não tendo havido tempo para uma maior expressão dos hormônios sexuais.

Cordeiros nascidos nos dois anos, 2001 e 2002, tiveram pesos similares ao nascimento e ao desmame (Tabelas 2 e 3), mostrando que os fatores ambientais não variaram muito entre os anos de avaliação.

Quando houve diferenças nos pesos ao desmame, estas foram devidas aos ganhos médios diários até o desmame. Onde na análise individual, o ganho médio diário do nascimento ao desmame apresentou uma diferença altamente significativa $(\mathrm{P}=0,0001)$ em favor dos cordeiros de partos simples. Por outro lado, quando se analisou os dados pela prole, cordeiros gêmeos apresentaram maior ganho de peso diário do nascimento ao desmame do que cordeiros de partos simples $(\mathrm{P}=0,0433)$.

Entre os grupos genéticos, observa-se na análise por prole que os cordeiros Suffolk, Hampshire Down e $1 / 2$ Corriedale $+1 / 2$ Texel apresentaram ganhos médios diários similares entre si, e superiores aos ganhos dos cordeiros Ile de France. Na análise individual os ganhos entre os grupamentos seguiram a mesma tendência, onde os Suffolk e Hampshire Down apresentaram os maiores ganhos e os Ile de France os menores. Os animais $1 / 2$ Corriedale $+1 / 2$ Texel apresentaram ganhos intermediários entre os Hampshire Down e os Ile de france, não diferindo destes.

Com relação a mortalidade dos cordeiros, podese observar (Tabela 4) que não houve diferença para todos os efeitos considerados (tratamento, sexo, idade da mãe, grupo genético e ano). Porém, quando se separou os cordeiros em únicos e gêmeos, dentro de cada efeito, foi observado diferenças entre os grupos genéticos na mortalidade de cordeiros únicos. Sendo que cordeiros únicos Hampshire Down e Ile de France apresentaram as menores taxas de mortalidade $(0,0 \%)$, sendo diferentes das mortalidades dos $1 / 2$ Corriedale $+1 / 2$ Texel $(20,0 \%)$ e dos Suffolk (25,0\%). Já para cordeiros gêmeos não se observou diferença entre os grupos genéticos.

Quando se comparou a mortalidade entre cordeiros gêmeos e únicos, observou-se que no geral, os gêmeos apresentaram maior mortalidade $(29,03 \%)$ do que os únicos (4,69\%). Segundo Minola e Goyenechea (1975), quando os valores de mortalidades de cordeiros do nascimento ao desmame é de até $13 \%$, pode ser considerado bom, acima de 13 até $27 \%$ é mediano, e acima de $27 \%$ é ruim. Estas médias gerais são reflexo do que ocorreu dentro de cada efeito. Para os tratamentos observou-se, que entre filhos de ovelhas que não 
receberam suplementação e entre filhos de ovelhas suplementadas com uma mistura de milho grão mais farelo de soja, uma maior mortalidade dos gêmeos comparado com os únicos. Esta mesma tendência ocorreu para o efeito de sexo, idade da mãe, ano e grupo genético, onde os cordeiros gêmeos apresentaram maior mortalidade. Porém, para grupo genético houve uma exceção onde cordeiros únicos de grupamento $1 / 2$ Corriedale + $1 / 2$ Texel apresentaram maior mortalidade $(20,0 \%)$ do que os gêmeos $(0,0 \%)$. Há de se ressaltar o pequeno número de cordeiros $1 / 2$ Corriedale $+1 / 2$ Texel e Suffolk, que pode ter exacerbado os dados de mortalidade nesses grupamentos genéticos.

Tabela 4. Número e taxa de mortalidade (percentagem entre parênteses) de cordeiros entre o nascimento e o desmame.

\begin{tabular}{|c|c|c|c|c|c|c|}
\hline \multirow{2}{*}{ Efeito } & \multirow{2}{*}{ Nascidos } & \multirow{2}{*}{ Mortalidade } & \multicolumn{2}{|c|}{ Únicos } & \multicolumn{2}{|c|}{ Gêmeos } \\
\hline & & & Nascidos & Mortos & Nascidos & Mortos \\
\hline \multicolumn{7}{|l|}{ Tratamento } \\
\hline $\mathrm{T} 1$ & 37 & $7(18,92)$ & 19 & $1(5,26) \mathrm{A}$ & 18 & $6(33,33) \mathrm{B}$ \\
\hline $\mathrm{T} 2$ & 46 & $6(13,04)$ & 20 & $1(5,00)$ & 26 & $5(19,23)$ \\
\hline $\mathrm{T} 3$ & 43 & $8(18,60)$ & 25 & $1(4,00) \mathrm{A}$ & 18 & $7(38,89) \mathrm{B}$ \\
\hline \multicolumn{7}{|l|}{ Sexo } \\
\hline Macho & 70 & $15(21,43)$ & 29 & $2(6,90) \mathrm{A}$ & 41 & $13(31,71) \mathrm{B}$ \\
\hline Fêmea & 56 & $6(10,71)$ & 35 & $1(2,86) \mathrm{A}$ & 21 & $5(23,81) \mathrm{B}$ \\
\hline \multicolumn{7}{|l|}{ Idade } \\
\hline 4 dentes & 25 & $4(16,00)$ & 17 & $2(11,76)$ & 8 & $2(25,00)$ \\
\hline 6 dentes & 27 & $2(7,41)$ & 17 & $0(0,00) \mathrm{A}$ & 10 & $2(20,00) \mathrm{B}$ \\
\hline 8 dentes & 74 & $15(20,27)$ & 30 & $1(3,33) \mathrm{A}$ & 44 & $14(31,82) \mathrm{B}$ \\
\hline \multicolumn{7}{|l|}{ Grupo genético } \\
\hline $1 / 2$ Corriedale $+1 / 2$ Texel & 11 & $1(9,09)$ & 5 & $1(20,00) b B$ & 6 & $0(0,00) \mathrm{A}$ \\
\hline Hampshire Down & 73 & $9(12,33)$ & 39 & $0(0,00) \mathrm{aA}$ & 34 & $9(26,47) \mathrm{B}$ \\
\hline Ile de France & 26 & $5(19,23)$ & 12 & $0(0,00) \mathrm{aA}$ & 14 & $5(35,71) \mathrm{B}$ \\
\hline Suffolk & 16 & $6(37,50)$ & 8 & $2(25,00) b$ & 8 & $4(50,00)$ \\
\hline \multicolumn{7}{|l|}{ Ano } \\
\hline 2001 & 77 & $15(19,48)$ & 37 & $1(2,70) \mathrm{A}$ & 40 & $14(35,00) \mathrm{B}$ \\
\hline 2002 & 49 & $6(16,66)$ & 27 & $2(7,41)$ & 22 & $4(18,18)$ \\
\hline Total & 126 & $21(16,66)$ & 64 & $3(4,69) \mathrm{A}$ & 62 & $18(29,03) \mathrm{B}$ \\
\hline
\end{tabular}

a, b: Médias seguidas de letras diferentes, em colunas, para o mesmo efeito, diferem significativamente a 5\% de probabilidade. A, B: Médias seguidas de letras diferentes, em linhas, para o mesmo efeito, diferem significativamente a 5\% de probabilidade. T1: Ovelhas sem suplementação; T2: Ovelhas suplementadas com milho triturado; T3: Ovelhas suplementadas com farelo de soja e milho triturado (25:75).

Fonte: Elaboração dos autores.

Os dados de mortalidade diferem dos apresentados por Mexia et al. (2004), que não observaram diferença estatística entre cordeiros únicos e gêmeos. Sendo que as médias observadas pelos autores foram de 27,39 e 48,68\% para cordeiros únicos e gêmeos, muito maiores do que as observadas no presente estudo.
A maior mortalidade encontrada nos cordeiros nascidos como gêmeos provavelmente está relacionada ao menor peso ao nascimento destes cordeiros (Tabela 3). De acordo com Coimbra Filho (1997) para a máxima sobrevivência dos cordeiros, há para cada raça um peso considerado adequado, e que no geral situa-se entre 3,5 a $5,0 \mathrm{~kg}$. 
Quanto menores os pesos menor a probabilidade de sobrevivência, ou maior de mortalidade. Chniter et al. (2011) observaram taxas de mortalidade em cordeiros D'man, de acordo com o peso ao nascer, de $52,2 \%$ (1,5 $\mathrm{kg}$ ou menos), $17,7 \%$ (entre 1,5 a $2,5 \mathrm{~kg}$ ), 6,7\% (entre 2,5 e 3,5 kg) e 1,8\% (acima de $3,5 \mathrm{~kg}$ ). Estes resultados estão de acordo com os encontrados por Christley et al. (2003), que pesquisaram fatores que afetam a mortalidade em cordeiros em dois grupos de dados, um com 1339 cordeiros e outro com 3172 cordeiros, de diversas raças na Grã-Bretanha. Os autores observaram que o baixo peso ao nascimento, resultado principalmente de efeitos ambientais maternos, como baixa condição corporal da mãe, e gestações gemelares, é o principal fator a contribuir para a mortalidade dos cordeiros. Estes cordeiros além de terem menores reservas corporais para enfrentar as condições ambientais, possuem também menores concentrações de imunoglobulinas séricas.

Na Tabela 5 são apresentados dados de consumo e de custos (de cada ingrediente e o total) da suplementação alimentar antes e durante a estação de monta, onde os preços foram baseados em valores praticados na região de Londrina no mês de abril de 2013.

Tabela 5. Consumo e custos da suplementação alimentar antes e durante a estação de monta.

\begin{tabular}{lccc}
\hline \multirow{2}{*}{ Tratamento } & \multicolumn{2}{c}{ Suplementação } & \multirow{2}{*}{ Custo da suplementação (em R\$) } \\
\cline { 2 - 3 } & Milho $(\mathrm{kg})$ & Farelo de soja $(\mathrm{kg})$ & - \\
T1 & - & - & 511,18 \\
T2 & $1.345,20$ & - & 655,79 \\
\hline
\end{tabular}

T1: Sem suplementação; T2: Suplementação com milho triturado; T3: Suplementação com milho e farelo de soja. Fonte: Elaboração dos autores.

A Tabela 6 apresenta a produção de cordeiros e os custos da suplementação alimentar no módulo de 100 ovelhas; onde verificou-se que a suplementação com milho triturado, realizada nas condições experimentais apresentadas, resultou em perda econômica de R\$277,83. Sendo que aqui ainda não foram computados gastos extras como consumo de energia, frete e mão de obra. A suplementação com milho triturado e farelo de soja mostrou-se ainda mais anti-econômica. Portanto, a suplementação alimentar antes e durante a estação de monta em ovelhas de corte, apesar de elevar as taxas de parição ((ovelhas em reprodução/ovelhas paridas)*100) e índices de natalidade (cordeiros nascidos/ovelhas em reprodução), não possibilitou retorno econômico. As taxas de parição e os índices de natalidade foram, respectivamente, 82,$4 ; 86,8$ e $85,0 \%$, e 1,$1 ; 1,2$ e 1,1 cordeiros, para ovelhas sem suplementação, suplementadas com $600 \mathrm{~g}$ de milho grão, e suplementadas com $600 \mathrm{~g}$ de milho grão mais farelo de soja (MORI et al., 2006). 
Tabela 6. Receita bruta e final dos cordeiros produzidos nos três tratamentos alimentares (utilizando o módulo de 100 ovelhas).

\begin{tabular}{lcccc}
\hline Tratamento & $\begin{array}{c}\text { Peso vivo dos } \\
\text { cordeiros (kg) }\end{array}$ & Receita bruta $(\mathrm{R} \$)^{*}$ & $\begin{array}{c}\text { Custos de suplementação } \\
\text { alimentar (R\$) }\end{array}$ & Receita final (R\$)** \\
\hline T1 & $1.318,53$ & $5.933,39$ & - & $5.933,39$ \\
$\mathrm{~T} 2$ & $1.560,79$ & $7.023,56$ & $1.368,00$ & $5.655,56$ \\
$\mathrm{~T} 3$ & $1.431,00$ & $6.439,50$ & $1.755,00$ & $4.684,50$ \\
\hline
\end{tabular}

T1: Sem suplementação; T2: Suplementação com milho triturado; T3: Suplementação com milho e farelo de soja.

* Considerando $\mathrm{R} \$ 4,50$ por $\mathrm{kg}$ de peso vivo.

** Descontado somente custos com alimentação das ovelhas antes e durante a estação de monta.

Fonte: Elaboração dos autores.

Esses resultados mostram que para que ocorra vantagem econômica no uso do flushing alimentar, o aumento na percentagem de partos gemelares tem que ser substancial, e ao mesmo tempo a mortalidade dos cordeiros gêmeos não pode ser muito maior do que a dos cordeiros únicos, e os cordeiros gêmeos tem que apresentar um desenvolvimento corporal igual ou próximo dos cordeiros únicos. Também deve ser reforçado que o preço pago nos alimentos pode variar de ano para ano, o que pode também afetar a resposta econômica esperada.

\section{Conclusões}

O flushing alimentar antes e durante a monta não afeta, subsequentemente, o ganho de peso e peso dos cordeiros. Por outro lado, possibilita aumento na produção total de quilos de cordeiros produzidos, porém, quando os custos de suplementação são elevados, torna a prática inviável economicamente.

\section{Agradecimentos}

Os autores agradecem ao suporte financeiro da Fundação Araucária e do $\mathrm{CNPq}$, bem como das bolsas de produtividade em pesquisa de Edson Luis de Azambuja Ribeiro, Ivone Yurika Mizubuti e Elzânia Sales Pereira.

\section{Referências}

BRAMLEY, P. S.; DENEHY, H. L.; NEWTON, J. E. The effect of different planes of nutrition before mating on the reproductive performance o Masham ewes. Veterinary Record, London, v. 99, n. 15, p. 294-296, 1976.

BRANCA, A.; MOLLE, G.; SITZIA, M.; DECANDIA, M.; LANDAU, S. Short-term dietary effects on reproductive wastage after induced ovulation and artificial insemination in primiparus lactating Sarda ewes. Animal Reproduction Science, Amsterdan, v. 58, n. 1, p. 59-71, 2000.

CASTRO, F. A. B.; RIBEIRO, E. L. A.; KORITIAKI, N. A.; MIZUBUTI, I. Y.; SILVA, L. D. F.; PEREIRA, E. S.; PINTO, A. P.; CONSTANTINO, C.; FERNANDES JUNIOR, F. Desempenho de cordeiros Santa Inês do nascimento ao desmame filhos de ovelhas alimentadas com diferentes níveis de energia. Semina: Ciências Agrárias, Londrina, v. 33, n. 6, p. 3379-3388, 2012 b. Suplemento 2.

CASTRO, F. A. B.; RIBEIRO, E. L. A.; MIZUBUTI, I. Y.; SILVA, L. D. F.; BARBOSA, M. A. A. F.; MARSON, B.; GRANDIS, F. A.; FERNANDES JUNIOR, F.; PEREIRA, E. S. Energia dietética ao final da gestação e durante a lactação e desempenho de ovinos Santa Inês em sistema de acasalamento acelerado. Semina: Ciências Agrárias, Londrina, v. 34, n. 6, p. 4187-4202, 2013. Suplemento 2.

CASTRO, F. A. B.; RIBEIRO, E. L. A.; MIZUBUTI, I. Y.; SILVA, L. D. F.; BARBOSA, M. A. A. F.; SOUSA, C. L.; PAIVA, F. H. P.; KORITIAKI, N. A. Influence of pre and postnatal energy restriction on the productive performance of ewes and lambs. Revista Brasileira de Zootecnia, Viçosa, MG, v. 41, n. 4, p. 951-958, 2012a. 
CHNITER, M.; HAMMADIA, M.; KHORCHANIA, T.; KRIT, R.; LAHSOUMI, B.; SASSI, M. B.; NOWAKD, R.; HAMOUDAH, M. B. Phenotypic and seasonal factors influence birth weight, growth rate and lamb mortality in D'man sheep maintained under intensive management in Tunisian oases. Small Ruminant Research, Amsterdan, v. 99, n. 2-3, p. 166-170, 2011.

CHRISTLEY, R. M.; MORGAN, K. L.; PARKIN, T. D. H.; FRENCH, N. P. Factors related to the risk of neonatal mortality, birth-weight and serum immunoglobulin concentration in lambs in the UK. Preventive Veterinary Medicine, Amsterdan, v. 57, n. 4, p. 209-226, 2003.

COIMBRA FILHO, A. Técnicas de criação de ovinos. 2. ed. Guaíba: Agropecuária, 1997. 102 p.

KORITIAKI, N. A.; RIBEIRO, E. L. A.; SCERBO, D. C.; MIZUBUTI, I. Y.; SILVA, L. D. F.; BARBOSA, M. A. A. F.; SOUZA, C. L.; PAIVA, F. H. P. Fatores que afetam o desempenho de cordeiros Santa Inês puros e cruzados do nascimento ao desmame. Revista Brasileira de Saúde e Produção Animal, Salvador, v. 13, n. 1, p. 258-270, 2012.

KORITIAKI, N. A.; RIBEIRO, E. L. A.; MIZUBUTI, I. Y.; SILVA, L. D. F.; BARBOSA, M. A. A. F.; SCERBO, D. C.; MUNIZ, C. A. S. D.; FERNANDES JÚNIOR, F. Effect of environmental factors on performance of purebred and crossbred Santa Inês lambs from birth to 154 days of age. Revista Brasileira de Zootecnia, Viçosa, MG, v. 42, n. 2, p. 87-94, 2013.

LASSOUED, N.; REKIK, M.; MAHOUACHI, M.; HAMOUDA, M. B. The effect of nutrition prior to and during mating on ovulation rate, reproductive wastage, and lambing rate in three sheep breeds. Small Ruminant Research, Amsterdan, v. 52, n. 1-2, p. 117-125, 2004.

MAACK, R. Geografia física do estado do Paraná. 2. ed. Rio de Janeiro: José Olympio, 1981. 450 p.

MEXIA, A. A.; MACEDO, F. A. F.; ALCALDE, C. R.; SAKAGUTI, E. S.; MARTINS, E. N.; ZUNDT, M.; YAMAMOTO, S. M.; MACEDO, R. M. Desempenhos reprodutivo e produtivo de ovelhas Santa Inês suplementadas em diferentes fases da gestação. Revista Brasileira de Zootecnia, Viçosa, MG, v. 33, n. 3, p. 658667, 2004.

MINOLA, J.; GOYENECHEA, J. Praderas \& lanares: produccion ovina em alto nível. Montevideo: Hemisfério Sur, 1975.365 p.

MOHAMMADI, H.; SHAHRBABAK, M. M.; SHAHRBABAK, H. M. Genetic analysis of ewe productivity traits in Makooei sheep. Small Ruminant Research, Amsterdan, v. 107, n. 2-3, p. 105-110, 2012.
MORI, R. M.; RIBEIRO, E. L. A.; MIZUBUTI, I. Y.; ROCHA, M. A.; SILVA, L. D. F. Desempenho reprodutivo de ovelhas submetidas a diferentes formas de suplementação alimentar antes e durante a estação de monta. Revista Brasileira de Zootecnia, Viçosa, MG, v. 35, n. 3, p. 1122-1128, 2006. Suplemento 3.

NAQVI, S. M. K.; SEJIAN, V.; KARIM, S. A. Effect of feed flushing during summer season on growth, reproductive performance and blood metabolites in Malpura ewes under semiarid tropical environment. Tropical Animal Health and Production, Berlin, v. 45, n. 1, p. 143-148, 2012.

NOTTLE, M. B.; KLEEMANN, D.; GROSSER, T. I.; SEAMARK, R. F. Evaluation of a nutritional strategy to increase ovulation rate in Merino ewes mated in late spring-early summer. Animal Reproduction Science, Amsterdan, v. 47, n. 4, p. 255-261, 1997.

PEETERS, R.; KOX, G.; VAN ISTERDAEL, J. Environmental and maternal effects on early postnatal growth of lambs of different genotypes. Small Ruminant Research, Amsterdan, v. 19, n. 1, p. 45-53, 1996.

RODA, D. S.; OTTO, P. A. Efeito da suplementação alimentar pré-acasalamento na prolificidade de ovinos das raças Ideal e Corriedale. Boletim de Indústria Animal, Nova Odessa, v. 47, n. 2, p. 87-96, 1990.

SCARAMUZZI, R. J.; RADFORD, H. M. Factors regulating ovulation rate in the ewe. Journal of Reproduction and Fertility, Colchester, v. 69, n. 1, p. 353-367, 1983.

SCHANBACHER, B. D.; CROUSE, J. D.; FERRELL, C. L. Testosterone influences on growth, performance, carcass characteristics and composition of young market lambs. Journal of Animal Science, Champaign, v. 51, n. 3, p. 685-691, 1980.

SILVA, D. J.; QUEIRÓZ, A. C. Análise de alimentos: métodos químicos e biológicos. 3. ed. Viçosa: UFV, 2002. $235 \mathrm{p}$.

SORMUNEN-CRISTIAN, R.; JAUHIAINEN, L. Effect of nutritional flushing on the productivity of Finnish Landrace ewes. Small Ruminant Research, Amsterdan, v. 43, n. 1, p. 75-83, 2002.

STATISTICAL ANALYSIS SYSTEM INSTITUTE SAS. SAS INSTITUTE. SAS/STAT user's guide. Cary: SAS Institute, 1994. v. 2, 796 p.

ZISHIRI, O. T.; ClOETE, S. W. P.; OLIVIER, J. J.; DZAMA, K. Genetic parameters for growth, reproduction and fitness traits in the South African Dorper sheep breed. Small Ruminant Research, Amsterdan, v. 112, n. 1-3, p. 39-48, 2013. 
